\title{
Several generalizations on Wolstenholme Theorem
}

Yuyang Zhu*, Jing Huang, Lili Wang, and Ming Li

Department of electronic and information engineering, Bozhou University 236800, China

Abstract. This paper generalizes Wolstenholme theorem on two aspects. The first generalization is a parameterized form: let $p>k+2, k \geq 1, \forall t \in \mathbb{Z}$, then $\frac{(p t+p-1) !}{(p t) !} \sum_{m=0}^{k-1}(-1)^{m} \sum_{1 \leq i_{1}<\cdots<i_{k-m} \leq p-1} \frac{p^{k-(m+1)}}{\prod_{l=1}^{k-m}\left(p t+i_{l}\right)} \equiv 0\left(\bmod p^{k+1}\right)^{.}$

The second generalization is on composite number module:

Let $\frac{1}{a}$ be the $x$ in congruent equation $a x \equiv 1(\bmod m)(1 \leq x<m)$, if $m \geq 5$, then

$$
\begin{aligned}
& \sum_{\substack{k, m)=1, 1 \leq j \leq m}}\left(\frac{1}{k}\right)^{2} \equiv \frac{m}{6}\left[2 m \varphi(m)+\prod_{p \mid m}(1-p)\right](\bmod m) ; \\
& \sum_{\substack{(k, m)=1, 1 \leq j \leq m}}\left(\frac{1}{k}\right)^{3} \equiv \frac{m^{2}}{4}\left[m \varphi(m)+\prod_{p \mid m}(1-p)\right](\bmod m) ; \\
& \sum_{\substack{(k, m)=1, 1 \leq j \leq m}}\left(\frac{1}{k}\right)^{4} \equiv \frac{m}{30}\left[6 m^{3} \varphi(m)+10 m^{2} \prod_{p \mid m}(1-p)-\prod_{p \mid m}\left(1-p^{3}\right)\right](\bmod m) ; \\
& \sum_{\substack{(k, m)=1, 1 \leq j \leq m}}\left(\frac{1}{k}\right)^{r} \equiv m^{r} \sum_{d \mid m} \mu(d)\left(\frac{m}{d}\right)^{-r} \sum_{k=1}^{\frac{m}{d}} k^{r}(\bmod m) .
\end{aligned}
$$

Where $\varphi(x)$ is Euler function, $\mu(x)$ is Möbius function.

Keywords: Wolstenholme theorem, Congruent, Composite number module, Möbius transform.

\section{Introduction}

Congruence modulo theory has wide applications in computer science, coding theory, and information security. It has a famous Wolstenholme theorem: if $p>3$ is a prime number,

\footnotetext{
*Corresponding author: zhuyy@hfuu.edu.cn
} 
then the sum of $1+\frac{1}{2}+\frac{1}{3}+\cdots+\frac{1}{p-1}$ must have a numerator that can be evenly divided by $p^{2}$, or $\sum_{k=1}^{p-1} \frac{(p-1) !}{k} \equiv 0\left(\bmod p^{2}\right)$.

This theorem has important applications and has been closely watched for its generalization[1-8]. Early in 1889, Leudesdorf [5] generalized Wolstenholme theorem by applying Bauer congruence: let $s_{m}=\sum_{\substack{k=1 \\(k, m)=1}}^{m-1} \frac{1}{k}$ and $m>1$, if $2 \vdash m, 3 \vdash m$, then $s_{m} \equiv 0\left(\bmod m^{2}\right) ;$ if $2 \vdash m, 3 \mid m$, then $s_{m} \equiv 0\left(\bmod \frac{m^{2}}{3}\right)$. If $2|m, 3| m$, then $s_{m} \equiv 0\left(\bmod \frac{m^{2}}{6}\right)$. If $m=2^{\alpha}(\alpha \geq 2)$, then $s_{m} \equiv 0\left(\bmod \frac{m^{2}}{4}\right)$. In 1995, Zeng [8] gives a uniform expression: if $m \geq 5$, then $s_{m} \equiv-\frac{m^{2}}{12}\left(2 m \varphi\left(m+\prod_{p \mid m}(1-p)\right)\right)\left(\bmod m^{2}\right)$.

In the following, this theorem will be generalized in two ways: a. in the case of general composite modulo; $b$. in the case of the same theorem with arguments.

\section{Main results}

\subsection{Generalization in the case of composite modulo}

In the following Wolstenholme theorem will be generalized in the case of general composite number $m$.

Definition For $m>1$, if $(a, m)=1$, then let the solution $x$ of congruence equation $a x \equiv 1(\bmod m)(0 \leq x<m)$ be the inverse of a modulus $m$, namely $a^{-1}$ or $\frac{1}{a}$.

It can be observed that, if $(a, m)=(b, m)=1$, then

$$
(a b)^{-1} \equiv a^{-1} b^{-1}(\bmod m), \quad a^{-1}+b^{-1} \equiv(a b)^{-1}(a+b)(\bmod m) .
$$

Möbius transformation will be used to prove the following:

Theorem 2.1.1 If $m \geq 5$, then

(i) $\sum_{\substack{(k, m)=1, 1 \leq j \leq m}}\left(\frac{1}{k}\right)^{2} \equiv \frac{m}{6}\left[2 m \varphi(m)+\prod_{p \mid m}(1-p)\right](\bmod m)$;

(ii) $\sum_{\substack{(k, m)=1, 1 \leq j \leq m}}\left(\frac{1}{k}\right)^{3} \equiv \frac{m^{2}}{4}\left[m \varphi(m)+\prod_{p \mid m}(1-p)\right](\bmod m)$;

(iii) $\sum_{\substack{k, m)=1, 1 \leq j \leq m}}\left(\frac{1}{k}\right)^{4} \equiv \frac{m}{30}\left[6 m^{3} \varphi(m)+10 m^{2} \prod_{p \mid m}(1-p)-\prod_{p \mid m}\left(1-p^{3}\right)\right](\bmod m)$. 
Lemma 2.1.2 Let $s_{k}(n)=n^{-k} \sum_{j=1}^{n} j^{k}, s_{k}^{*}(n)=n^{-k} \sum_{\substack{(j, n)=1, 1 \leq j \leq n}} j^{k}, \quad$ then $\sum_{d \mid n} s_{k}^{*}(d)=s_{k}(n)$

Proof $s_{k}(n)=n^{-k} \sum_{j=1}^{n} j^{k}=n^{-k} \sum_{d \mid n} \sum_{\substack{(j, n)=d, 1 \leq j \leq n}} j^{k}=\sum_{d \mid n} n^{-k} \sum_{\substack{(j, n)=d, 1 \leq j \leq n}} j^{k}$

$$
=\sum_{d \mid n} n^{-k} \sum_{\substack{\left(t, \frac{n}{d}\right)=1, 1 \leq t \leq \frac{n}{d}}} t^{k} d^{k}=\sum_{d \mid n}\left(\frac{n}{d}\right)^{-k} \sum_{\substack{\left(t, \frac{n}{d}\right)=1, 1 \leq t \leq \frac{n}{d}}} t^{k}=\sum_{d \mid n} s_{k}^{*}\left(\frac{n}{d}\right)=\sum_{d \mid n} s_{k}^{*}(d)
$$

The lemma is proved.

Proof of theorem 2.1.1 For any $1 \leq i<j \leq m-1$ we have $\frac{1}{i} \not \neq \frac{1}{j}$, otherwise it leads to contradiction $0 \equiv \frac{1}{i}-\frac{1}{j} \equiv \frac{i-j}{i j}(\bmod m)$. Thus when $\frac{1}{i}$ iterates from 1 to $m-1,(i$, $m)=1, i$ also iterates from 1 to $m-1,(i, m)=1$, thus

$$
\sum_{\substack{(k, m)=1, 1 \leq j \leq m}}\left(\frac{1}{k}\right)^{2} \equiv \sum_{\substack{(k, m)=1, 1 \leq j \leq m}} k^{2}(\bmod m)
$$

Then according to lemma 2.1.2 and Möbius transformation,

$$
\begin{aligned}
\sum_{\substack{(k, m)=1, 1 \leq j \leq m}} k^{2} & =m^{2}\left(m^{-2} \sum_{\substack{(k, m)=1, 1 \leq j \leq m}} k^{2}\right)=m^{2} s_{2}^{*}(m)=m^{2} \sum_{d \mid m} \mu(d) s_{2}\left(\frac{n}{d}\right) \\
& =m^{2} \sum_{d \mid m} \mu(d) s_{2}\left(\frac{m}{d}\right)=m^{2} \sum_{d \mid m} \mu(d)\left(\frac{m}{d}\right)^{-2} \frac{1}{6} \cdot \frac{m}{d}\left(\frac{m}{d}+1\right)\left(\frac{2 m}{d}+1\right) \\
& =m^{2} \sum_{d \mid m} \mu(d)\left(\frac{m}{3 d}+\frac{1}{2}+\frac{d}{6 m}\right)=m^{2}\left(\frac{1}{3} \varphi(m)+0+\frac{1}{6 m} \prod_{p \mid m}(1-p)\right) \\
& =m\left(\frac{m \varphi(m)}{3}+\frac{1}{6} \prod_{p \mid m}(1-p)\right)=\frac{m}{6}\left(2 m \varphi(m)+\prod_{p \mid m}(1-p)\right)
\end{aligned}
$$

thus

$$
\sum_{\substack{(k, m)=1, 1 \leq j \leq m}}\left(\frac{1}{k}\right)^{2} \equiv \sum_{\substack{k, m)=1, 1 \leq j \leq m}} k^{2} \equiv \frac{m}{6}\left(2 m \varphi(m)+\prod_{p \mid m}(1-p)\right)(\bmod m) .
$$

Hence (i) is proved.

For the same reason

$$
\sum_{\substack{(k, m)=1, 1 \leq j \leq m}}\left(\frac{1}{k}\right)^{3} \equiv \sum_{\substack{(k, m)=1, 1 \leq j \leq m}} k^{3}(\bmod m)
$$

Also according to lemma 2.1.2 and Möbius transformation, 


$$
\begin{aligned}
\sum_{\substack{k, m)=1, 1 \leq j \leq m}} k^{3} & =m^{3}\left(m^{-3} \sum_{\substack{k, m)=1, 1 \leq j \leq m}} k^{3}\right)=m^{3} s_{3}^{*}(m)=m^{3} \sum_{d \mid m} \mu(d) s_{3}\left(\frac{n}{d}\right) \\
& =m^{3} \sum_{d \mid m} \mu(d) s_{3}\left(\frac{m}{d}\right)=m^{3} \sum_{d \mid m} \mu(d)\left(\frac{m}{d}\right)^{-3} \frac{1}{4}\left(\frac{m}{d}\right)^{2}\left(\frac{m}{d}+1\right)^{2} \\
& =m^{3} \sum_{d \mid m} \mu(d)\left(\frac{m}{4 d}+\frac{1}{2}+\frac{d}{4 m}\right)=m^{2}\left(\frac{1}{4} \varphi(m)+0+\frac{1}{4 m} \prod_{p \mid m}(1-p)\right) \\
& =m^{2}\left(\frac{m \varphi(m)}{4}+\frac{1}{4} \prod_{p \mid m}(1-p)\right)=\frac{m^{2}}{4}\left(m \varphi(m)+\prod_{p \mid m}(1-p)\right) .
\end{aligned}
$$

Thus

$$
\sum_{\substack{(k, m)=1, 1 \leq j \leq m}}\left(\frac{1}{k}\right)^{3} \equiv \sum_{\substack{(k, m)=1, 1 \leq j \leq m}} k^{3} \equiv \frac{m^{2}}{4}\left(m \varphi(m)+\prod_{p \mid m}(1-p)\right)(\bmod m) .
$$

Therefore, (ii) is proved. (iii) can be proved in a similar way.

From the proof of theorem 2.1.1, for any natural number $r$, we can conclude:

Theorem 2.1.3 If $m \geq 5, r(\geq 2)$ being a natural number, then

$$
\sum_{\substack{(k, m)=1, 1 \leq j \leq m}}\left(\frac{1}{k}\right)^{r} \equiv m^{r} \sum_{d \mid m} \mu(d) s_{r}\left(\frac{n}{d}\right) \equiv m^{r} \sum_{d \mid m} \mu(d)\left(\frac{m}{d}\right)^{-r} \sum_{k=1}^{\frac{m}{d}} k^{r}(\bmod m) .
$$

\subsection{Generalization in a parameter form}

Theorem 2.2.1 Let prime number $p>k+2, k \geq 1, \forall t \in \mathbb{Z}$, then

$$
\frac{(p t+p-1) !}{(p t) !} \sum_{m=0}^{k-1}(-1)^{m} \sum_{1 \leq i_{1}<\cdots<i_{k-m} \leq p-1} \frac{p^{k-(m+1)}}{\prod_{l=1}^{k-m}\left(p t+i_{l}\right)} \equiv 0\left(\bmod p^{k+1}\right) .
$$

Lemma 2.2.2 ([7]) Let the number of solutions for congruence equation:

$$
f(x)=a_{n} x^{n}+\cdots+a_{1} x+a_{0} \equiv 0(\bmod p)
$$

Be greater than $n$, where $p$ is a prime, and $a_{i}(i=0,1,2, \cdots, n)$ is an integer. Then

$$
p \mid a_{i}(i=0,1, \cdots, n) .
$$

Lemma 2.2.3 For any prime number $p, \forall t \in \mathbb{Z}$, the coefficients of polynomial

$$
f(x)=[x-(t p+1)][x-(t p+2)] \cdots[x-(t p+p-1)]-x^{p-1}+1
$$

Can be evenly divided by $p$.

Proof Let $g(x)=[x-(t p+1)][x-(t p+2)] \cdots[x-(t p+p-1)], \quad$ then $1,2, \cdots, p-1$ are the solutions of the congruence equation

$$
g(x) \equiv 0(\bmod p) .
$$

According to Fermat's little theorem, $1,2, \cdots, p-1$ are also the solutions of congruence equation 


$$
h(x)=x^{p-1}-1 \equiv 0(\bmod p) .
$$

Thus there are $p-1$ solutions of the congruence equation

$$
f(x)=g(x)-h(x)(\bmod p),
$$

where $f(x)$ is a $p-2$ th order polynomial. According to lemma 2.2.2, all its coefficients can be evenly divided by $p$. The theorem is proved.

Proof of theorem 2.2.1 Let

$$
\begin{aligned}
g(x) & =[x-(t p+1)][x-(t p+2)] \cdots[x-(t p+p-1)] \\
& =x^{p-1}-s_{1} x^{p-2}+s_{2} x^{p-3}+\cdots-s_{p-2} x+\frac{(p t+p-1) !}{(p t) !} .
\end{aligned}
$$

where $s_{j}(j=1,2, \cdots, p-2)$ is an integer, and

$$
s_{p-m}=\sum_{1 \leq i_{1}<\cdots<i_{m-1} \leq p-1} \frac{1}{\left(p t+i_{1}\right)\left(p t+i_{2}\right) \cdots\left(p t+i_{m-1}\right)} \cdot \frac{(p t+p-1) !}{(p t) !}
$$

where $m=2,3, \cdots, p-1$. According to lemma 2.2.3, $p \mid s_{j}(j=1,2, \cdots, p-2)$. Let $x=(2 t+1) p$ in (1), since $g[(2 t+1) p]=\frac{(p t+p-1) !}{(p t) !}$, according to $(1)$,

$$
\frac{(p t+p-1) !}{(p t) !}=[(2 t+1) p]^{p-1}-s_{1}[(2 t+1) p]^{p-2}+\cdots-s_{p-2}[(2 t+1) p]+\frac{(p t+p-1) !}{(p t) !} .
$$

Hence

$$
[(2 t+1) p]^{p-1}-s_{1}[(2 t+1) p]^{p-2}+\cdots-s_{p-2}[(2 t+1) p]=0 .
$$

Thus

$$
\begin{aligned}
& {[(2 t+1) p]^{k}\left\{[(2 t+1) p]^{p-k-1}-s_{1}[(2 t+1) p]^{p-k-2}+\cdots+(-)^{k-1} s_{p-k-1}\right\}} \\
& \quad+(-1)^{k}[(2 t+1) p]^{k-1} s_{p-k}+\cdots+[(2 t+1) p]^{2} s_{p-3}-[(2 t+1) p] s_{p-2}=0 .
\end{aligned}
$$

Since $p>k+2, k \geq 1, p \mid s_{j}(j=1,2, \cdots, p-2)$,

$[(2 t+1) p]^{k}\left\{[(2 t+1) p]^{p-k-1}-s_{1}[(2 t+1) p]^{p-k-2}+\cdots+(-)^{k-1} s_{p-k-1}\right\} \equiv 0\left(\bmod p^{k+1}\right)$.

According to (3) and (4),

$$
(-1)^{k}[(2 t+1) p]^{k-1} s_{p-k}+\cdots+[(2 t+1) p]^{2} s_{p-3}-[(2 t+1) p] s_{p-2} \equiv 0\left(\bmod p^{k+1}\right) .
$$

Theorem 2.2.1 is proved by (5) and (2).

Let $t=0$ in theorem 2.2.1, then

Inference 2.2.4 Let prime number $p>k+2$, then

$$
(p-1) ! \sum_{m=0}^{k-1}(-1)^{m} \sum_{1 \leq i_{1}<\cdots<i_{k-m} \leq p-1} \frac{p^{k-(m+1)}}{\prod_{s=1}^{k-m} i_{s}} \equiv 0\left(\bmod p^{k+1}\right) .
$$

In theorem 2.2.1, $t=0, k=1$ is the case of Wolstenholme theorem. An extra parameter $t$ makes the theorem more applicable and more convenient to use.

This project is supported by the natural science foundation of Bozhou University. 


\section{References}

1. D.F.Bailey. Two p3 Variations of Lucas' Theorem[J]. Journal of number theory. 35(1990): 208-215.

2. Jianqiang Zhao. Bernoulli numbers, Wolstenholme's theorem, and $p 5$ variations of Lucas' theorem[J]. Journal of number theory. 123(2007), 18-26.

3. Romeo Meštrovic', Kotor. A note on the congruence $\left(\begin{array}{l}n p^{k} \\ m p^{k}\end{array}\right) \equiv\left(\begin{array}{l}n \\ m\end{array}\right)\left(\bmod p^{r}\right)[\mathrm{J}]$. Czechoslovak Mathematical Journal, 62 (137) (2012): 59-65.

4. Romeo Meštrovic', Kotor. Congruences for Wolstenholme primes[J]. Czechoslovak Mathematical Journal, 65 (140) (2015): 237-253.

5. G. H. Hardy, and E. M. Wright. An introduction to the theory of numbers (Fifth edition), Oxford University Press.1981: 88-104.

6. P. Ribenboim. (Translated by Sun Shuling and Feng keqin). Broad and profound prime number. Science Press, 2007,1:20-21.

7. Zhao Ke, Qi Sun. Lecture notes on number theory (1). Higher education press.1986.3: 44-45.

8. Denggao Zeng. The generalization of Wolstenholme theorem. Mathematics in practice and theory, 1995, 3: 89-91. 